ment $a$ which suffice. Let $F=B(t), B$ any field of characteristic two and $t$ transcendental over $B$. If $f(t)$ denotes an arbitrary element of $B(t)$, then define $\alpha$ by $f(t) \alpha=f(1 / t)$, and let $a=t+1 / t$.

\title{
BIBLIOGRAPHY
}

1. R. H. Bruck and Erwin Kleinfeld, The structure of alternative division rings, Proc. Amer. Math. Soc. vol. 2 (1951) pp. 878-890.

2. Marshall Hall, Projective planes, Trans. Amer. Math. Soc. vol. 54 (1943) pp. 229-277.

3. L. A. Skornyakov, Right alternative fields, Bull. Acad. Sci. URSS. Sér. Math. vol. 15 (1951) pp. 177-184.

The UNIVERSITY OF Oregon

\section{ON THE CHARACTERISTIC FUNCTION OF A MATRIX PRODUCT}

\section{S. GODDARD}

In a recent note [1], Roth has proved this result.

Theorem 1. Let $A$ and $B$ be $n \times n$ matrices, with elements in a field $F$, and let

$$
|x I-A|=a_{0}\left(x^{2}\right)-x a_{1}\left(x^{2}\right), \quad|x I-B|=b_{0}\left(x^{2}\right)-x b_{1}\left(x^{2}\right),
$$

where $a_{0}, a_{1}, b_{0}$, and $b_{1}$ are elements in the polynomial ring $F[x]$. If the rank of $A-B$ is not greater than unity, then

$$
|x I-A B|=(-)^{n}\left[a_{0}(x) b_{0}(x)-x a_{1}(x) b_{1}(x)\right] \text {. }
$$

In his proof, which is essentially a verification, Roth derives some interesting but unnecessary information. Here I present a proof which is shorter, direct, and leads naturally to a more general result involving three matrices.

The essential step in my proof is the observation that if $A$ is a nonsingular matrix and $M$ is a matrix of rank 1 , then

$$
|A+M|=|A|+\sum \Delta_{i}
$$

where $\sum \Delta_{i}$ is a sum of $n$ determinants, each consisting of $n-1$ columns of $A$ and one column of $M$. This follows from the fact that, $M$ being of rank 1, any two columns of $M$ are linearly dependent.

Received by the editors June 25, 1954. 
For the case at hand we have

$$
\begin{aligned}
|x I-A||x I+B| & =|(x I-A)(x I+B)| \\
& =\left|x^{2} I-A B-x(A-B)\right|
\end{aligned}
$$

and this determinant is equal to $\left|x^{2} I-A B\right|$ if $A-B$ has zero rank, while if $A-B$ has rank 1, we have

$$
\left|x^{2} I-A B-x(A-B)\right|=\left|x^{2} I-A B\right|-x \sum \Delta_{i},
$$

where each determinant $\Delta_{i}$ has $n-1$ columns chosen from $x^{2} I-A B$ and one column from $A-B$. It is observed that the terms of $x \sum \Delta_{i}$ contain only odd powers of $x$. Thus, in either case, $\left|x^{2} I-A B\right|$ is equal to the even part of $|x I-A||x I+B|$. Now

$$
|x I-A||x I+B|=(-)^{n}\left[a_{0}\left(x^{2}\right)-x a_{1}\left(x^{2}\right)\right]\left[b_{0}\left(x^{2}\right)+x b_{1}\left(x^{2}\right)\right] \text {, }
$$

and the even part is $(-)^{n}\left[a_{0}\left(x^{2}\right) b_{0}\left(x^{2}\right)-x^{2} a_{1}\left(x^{2}\right) b_{1}\left(x^{2}\right)\right]$. Hence, writing $y=x^{2}$, we have

$$
|y I-A B|=(-)^{n}\left[a_{0}(y) b_{0}(y)-y a_{1}(y) b_{1}(y)\right],
$$

and this is Roth's result.

Before extending this result we prove the

Lemma. If $H$ and $K$ are nonzero square matrices, such that $x H-K$ is of rank 1 , for $x$ indeterminate over the field $F$, then either

(i) $H=u^{\prime} \boldsymbol{h}^{\prime}, K=\mathbf{u} \boldsymbol{k}^{\prime}$, or

(ii) $H=\boldsymbol{u} \boldsymbol{h}^{\prime}, K=\boldsymbol{v} \boldsymbol{h}^{\prime}$, where $u, v, h, k$ are column vectors. Conversely, if $H$ and $K$ satisfy (i) and (ii) then $x H-K$ is of rank 1.

Proof. Since $x H-K$ is of rank 1 for all $x$, it follows that $H$ and $K$ are each of rank 1 and hence are of the form

$$
H=u^{\prime}, \quad K=v k^{\prime},
$$

where $u, v, h, k$ are column vectors. If we now equate to zero all the two-rowed minors of $x H-K$, it is easily found that either $u=v$ or $\boldsymbol{h}=\boldsymbol{k}$, and this proves the lemma. The converse is obviously true.

From this lemma we proceed to

Theorem 2. Let $A_{1}, A_{2}$, and $A_{3}$ be $n \times n$ matrices, such that

$$
\left|x I-A_{i}\right|=a_{0 i}\left(x^{3}\right)+x a_{1 i}\left(x^{3}\right)+x^{2} a_{2 i}\left(x^{3}\right) \quad(i=1,2,3)
$$

and write $H=A_{1}+A_{2}+A_{3}, K=A_{1} A_{2}+A_{1} A_{3}+A_{2} A_{3}$. If $H$ and $K$ satisfy the lemma, or if $H=K=0$, then 


$$
\begin{aligned}
\left|x I-A_{1} A_{2} A_{3}\right|= & a_{01} a_{02} a_{03}+x\left[a_{11}\left(a_{02} a_{23}+a_{03} a_{22}\right)\right. \\
& \left.+a_{12}\left(a_{01} a_{23}+a_{03} a_{21}\right)+a_{13}\left(a_{01} a_{22}+a_{02} a_{21}\right)\right] \\
& +x^{2} a_{21} a_{22} a_{23},
\end{aligned}
$$

where $a_{i j}=a_{i j}(x)$.

Proof. We have

$$
\left(x I-A_{1}\right)\left(x I-A_{2}\right)\left(x I-A_{3}\right)=x^{3} I-A_{1} A_{2} A_{3}-x(x H-K) .
$$

If $H=K=0$ we have

$$
E \equiv\left|x I-A_{1}\right|\left|x I-A_{2}\right|\left|x I-A_{8}\right|=\left|x^{8} I-A_{1} A_{2} A_{8}\right| .
$$

If $x H-K$ is of rank 1 for all $x$, we have

$$
E=\left|x^{8} I-A_{1} A_{2} A_{3}\right|-x \sum \Delta_{i},
$$

where each determinant $\Delta_{i}$, since it consists of $n-1$ columns of $x^{3} I-A_{1} A_{2} A_{3}$ and 1 column of $x H-K$, expands into a polynomial each term of which involves $x$ to the power $3 k$ or $3 k+1$ for some integer $k$. Now $x \sum \Delta_{i}$ is a polynomial, each term of which involves $x$ to a power $3 k+1$ or $3 k+2$. Thus, in either case, $\left|x^{3} I-A_{1} A_{2} A_{3}\right|$ is equal to the sum of the terms of $\left|x I-A_{1}\right|\left|x I-A_{2}\right|\left|x I-A_{3}\right|$ which involve powers of $x^{3}$. If we pick out these terms and replace $x^{3}$ by $x$ the result follows.

\section{REFERENCE}

W. E. Roth, On the characteristic polynomial of the product of two matrices, Proc. Amer. Math. Soc. vol. 5 (1954) pp. 1-3.

King's College, Aberdeen 